\title{
Cyclic nucleotide binding and structural changes in the isolated GAF domain of Anabaena adenylyl cyclase, CyaB2
}

Kabir H Biswas, Suguna Badireddy, Abinaya Rajendran, Ganesh S Anand, Sandhya S Visweswariah

GAF domains are a large family of regulatory domains, and a subset are found associated with enzymes involved in cyclic nucleotide (cNMP) metabolism such as adenylyl cyclases and phosphodiesterases. CyaB2, an adenylyl cyclase from Anabaena, contains two GAF domains in tandem at the N-terminus and an adenylyl cyclase domain at the C-terminus. Cyclic AMP, but not CGMP, binding to the GAF domains of CyaB2 increases the activity of the cyclase domain leading to enhanced synthesis of CAMP. Here we show that the isolated GAFb domain of CyaB2 can bind both CAMP and CGMP, and enhanced specificity for CAMP is observed only when both the GAFa and the GAFb domains are present in tandem (GAFab domain). In silico docking and mutational analysis identified distinct residues important for interaction with either CAMP or CGMP in the GAFb domain. Structural changes associated with ligand binding to the GAF domains could not be detected by bioluminescence resonance energy transfer (BRET) experiments. However, amide hydrogen-deuterium exchange mass spectrometry (HDXMS) experiments provided insights into the structural basis for CAMP-induced allosteric regulation of the GAF domains, and differences in the changes induced by CAMP and CGMP binding to the GAF domain. Thus, our findings could allow the development of molecules that modulate the allosteric regulation by GAF domains present in pharmacologically relevant proteins. 


\section{Cyclic nucleotide binding and structural changes in the isolated GAF domain of}

3 Anabaena adenylyl cyclase, CyaB2

4 Authors: Kabir Hassan Biswas ${ }^{1,3}$, Suguna Badireddy ${ }^{2}$, Abinaya Rajendran ${ }^{1}$, Ganesh Srinivasan Anand ${ }^{2}$

5 and Sandhya S Visweswariah ${ }^{1}$

6 Affiliations:

$7{ }^{1}$ Department of Molecular Reproduction, Development and Genetics, Indian Institute of Science,

8 Bangalore - 560012, India;

92 Department of Biological Sciences, National University of Singapore, Singapore - 117543, Singapore

$10{ }^{3}$ Current affiliation: Mechanobiology Institute, National University of Singapore, Singapore 117411,

11 Singapore

\section{Corresponding authors:}

13 Sandhya S Visweswariah, Department of Molecular Reproduction, Development and Genetics, Indian

14 Institute of Science, Bangalore - 560012, India, Tel: +91 80 22932542/23601522, email:

15 sandhya@mrdg.iisc.ernet.in

16 Kabir Hassan Biswas, Mechanobiology Institute, National University of Singapore, Singapore 117411,

17 Singapore, Tel: +65 66018983, email: mbikhb@,nus.edu.sg

19 Keywords: BRET; cAMP; cGMP; cyclases; GAF; HDXMS; ligand; phosphodiesterases; structural 20 changes 
21 Abbreviations: BRET, bioluminescence resonance energy transfer; cAMP, adenosine 3', 5' -cyclic

22 monophosphate; cGMP, guanosine 3', 5' -cyclic monophosphate; GAF, cGMP-specific and -regulated

23 cyclic nucleotide phosphodiesterase, Adenylyl cyclase, and E. coli transcription factor FhlA; HDXMS,

24 amide hydrogen/deuterium exchange mass spectrometry;

26 ABSTRACT

27 GAF domains are a large family of regulatory domains, and a subset are found associated with

28 enzymes involved in cyclic nucleotide (cNMP) metabolism such as adenylyl cyclases and

29 phosphodiesterases. CyaB2, an adenylyl cyclase from Anabaena, contains two GAF domains in

30 tandem at the $\mathrm{N}$-terminus and an adenylyl cyclase domain at the $\mathrm{C}$-terminus. Cyclic AMP, but not

31 cGMP, binding to the GAF domains of CyaB2 increases the activity of the cyclase domain leading to

32 enhanced synthesis of cAMP. Here we show that the isolated GAFb domain of CyaB2 can bind both

33 cAMP and cGMP, and enhanced specificity for cAMP is observed only when both the GAFa and the

$34 \mathrm{GAFb}$ domains are present in tandem (GAFab domain). In silico docking and mutational analysis

35 identified distinct residues important for interaction with either cAMP or cGMP in the GAFb domain.

36 Structural changes associated with ligand binding to the GAF domains could not be detected by

37 bioluminescence resonance energy transfer (BRET) experiments. However, amide hydrogen-deuterium

38 exchange mass spectrometry (HDXMS) experiments provided insights into the structural basis for

39 cAMP-induced allosteric regulation of the GAF domains, and differences in the changes induced by

40 cAMP and cGMP binding to the GAF domain. Thus, our findings could allow the development of

41 molecules that modulate the allosteric regulation by GAF domains present in pharmacologically

42 relevant proteins. 

INTRODUCTION

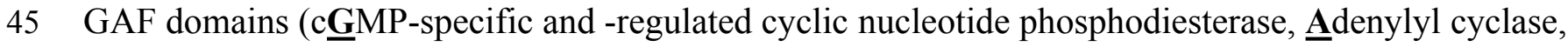

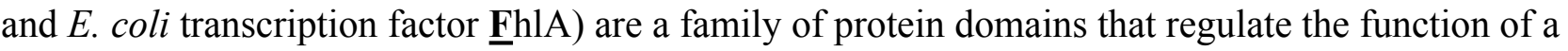
variety of domains with which they are associated (Aravind \& Ponting 1997; Charbonneau et al. 1990). They represent one of the largest families of small molecule-binding regulatory domains, and are found in organisms in all three kingdoms of life (Anantharaman et al. 2001; Martinez et al. 2002a). GAF domains ( $\sim 150$ amino acids long) are found associated with additional signaling domains such as the PAS, Sigma54_activat, helix-turn-helix (HTH), PEP_utilizers_C, GGDEF, EAL, HisKA and phosphodiesterase domains (Aravind \& Ponting 1997; Finn et al. 2010). GAF domains can bind a variety of ligands including tetrapyrroles, formate, haeme, bilin and cyclic nucleotides (Anantharaman et al. 2001; Zoraghi et al. 2004). Although the sequences of these domains have diverged substantially due to their long evolutionary history (Aravind et al. 2002), a motif of five residues (NKFDE) is conserved in most of the characterized cNMP-binding GAF domains (Zoraghi et al. 2004).

The structures of a number of cNMP-binding GAF domains have been solved by X-ray crystallography and NMR. These include the GAF domains in the cGMP-stimulated, cAMP phosphodiesterase, PDE2 [PDB: 1MC0] (Martinez et al. 2002b), Anabaena CyaB2 adenylyl cyclase [PDB: 1YKD] (Martinez et al. 2005) and the cGMP-stimulated, cGMP-specific PDE5 [PDB: 2K31, 3IBJ, 2ZMF, 3FLV] (Heikaus et al. 2009; Pandit et al. 2009; Russwurm et al. 2011; Wang et al. 2010). A common structural feature shared by these GAF domain is the presence of six central anti-parallel $\beta$-sheets flanked by $\alpha$-helices on both sides (Heikaus et al. 2009). The $\beta$-sheets form a curved plane that separates the $\alpha$-helices into two groups. The curved plane of the antiparallel $\beta$-sheets serves as the base of the ligand-binding pocket, and the rest of the ligand-binding pocket is covered by helices $\alpha 3, \alpha 4$, and some loop regions. Helices $\alpha 2$ and $\alpha 5$ are present on the opposite side of the ligand-binding pocket. In CyaB2, helix $\alpha 2$ 
connects the GAFb domain to N-terminal GAFa domain in CyaB2 and helix $\alpha 5$ connects the GAF domain to the C-terminal PAS and adenylyl cyclase effector domains. Cyclic AMP is buried within the ligand-binding pocket (Fig. 1A), and important residues in the ligand binding pocket that interact with cAMP include Arg 291 (H-bond with N1 of cAMP), Thr 293 (H-bond with N6 and N7 of cAMP), Asp 356 and Asn 359 (water mediated H-bond with N3 of cAMP), and Ile 308 (hydrophobic contact to the adenine ring of cAMP).

Most of the cNMP binding GAF domains show high specificity towards a specific cyclic nucleotide, but the basis for this selectivity in some GAF domains still remains unknown. Substitution of a region of the $\mathrm{CyaB} 1 \mathrm{GAFb}$ domain with that of a corresponding region in the cGMP-binding GAF domain of PDE2, allowed CyaB1 to show cGMP-enhanced adenylyl cyclase activity. However, the converse experiment in which amino acids in the PDE2 GAF domain were replaced with those from CyaB1 did not lead to altered specificity (Linder et al. 2007). In addition, structural studies combined with mutational analysis of the GAFa domain of PDE5 suggested that a key residue (Asp 164) allowed the discrimination between cAMP and cGMP (Heikaus et al. 2008).

In the present study, we show by direct ligand binding assays that the specificity of nucleotide binding 3 is reduced in an isolated GAF domain, as compared to the tandem GAFab domains of CyaB2. In silico docking and mutation of key interacting residues provided insights on cGMP binding, and HDXMS identified diverse structural changes induced by cAMP and cGMP.

\section{Generation of various GAF domain constructs and mutagenesis}


89 The nucleotide sequence of the GAFb domain of CyaB2 from Anabaena sp. PCC 7120 spanning

90 residues L270 to L431 was amplified by PCR from the full-length CyaB2 gene cloned into pQE30

91 plasmid (pQE30-CyaB2 (Bruder et al. 2005)) using primers GAFbf793 (5'

92 CTGGGATCCGGTACCCTGGATTTAGAAGATACCC 3') and GAFbr1279 (5'

93 ACACTCGAGCGATATCTAAAGCCACCCCGGC 3'). The PCR product was directly cloned into

94 pGEM-T-Easy vector (Promega) to generate the plasmid pGEM-T-GAFb and insert was sequenced. To

95 generate a GST fusion protein for cyclic nucleotide binding experiments and His $_{6}$-tagged protein for

96 HDXMS experiments, the GAFb nucleotide sequence was released and subcloned into pGEX-6p-1

97 plasmid vector (GE Healthcare) and pPRO-Ex-B plasmid vector (Invitrogen), respectively, using

98 BamHI and XhoI sites, resulting in the pGEX-6p-1-GAFb and pPRO-Ex-GAFb plasmid. To generate a

99 BRET-based sensor, the GAFb gene fragment was released and subcloned into the pGFP $^{2}$-MCS-Rluc

100 plasmid vector (PerkinElmer Life Sciences) using KpnI and EcoRV sites, resulting in the pGFP2-

$101 \mathrm{GAFb}-\mathrm{Rluc}$ plasmid. The I308A and T293A mutations in the GAFb domain was introduced using a

102 single primer (Shenoy \& Visweswariah 2003) and primers GAFb_CyaB2(I308A) (5'

103 GGACGAAAGCTACCCAAGATAATGGTTCTACTAAGG 3') and GAFb_CyaB2(T293A).

104 (5'GATGAACGCGGACCGCAGTGCCTTATGGCTGATAG 3'). Mutations were confirmed by

105 sequencing.

106 The nucleotide sequence of the tandem GAFab domains of CyaB2 spanning residues S77 to L431 was

107 amplified by PCR from the full-length CyaB2 gene cloned into pQE30 plasmid (pQE30-CyaB2

108 (Bruder et al. 2005)) using primers GAFaf216 (5'

109 GTCAAtgTtGgGAtCCCACGGTACCGAAAATATCCTGC 3') and GAFbr 1279. The PCR

110 product was directly cloned into pGEM-T-Easy vector (Promega) and sequenced. To express a GST

111 fusion protein, the tandem GAFab domain gene fragment was subcloned into pGEX-6p-1 plasmid 
112 vector (GE Healthcare) using BamHI and XhoI sites, resulting in the pGEX-6p-1-GAFab plasmid. To

113 generate a BRET-based sensor construct, the tandem GAFab domain gene fragment was subcloned

114 into the pGFP'2-MCS-Rluc plasmid vector (PerkinElmer Life Sciences) using EcoRV and XhoI sites,

115 resulting in the generation of pGFP2 - GAFab-Rluc plasmid.

116 The nucleotide sequence encoding residues M1 to L431 was PCR amplified from the full-length

117 CyaB2 gene cloned into pQE30 plasmid (pQE30-CyaB2 (Bruder et al. 2005)) using primers

118 GAFab_CyaB2f1 (5'ATATGGATCCGGTACCATGTCATTGCAACAGCG 3') and

119 GAFb_CyaB2r1271 (5'ACACTCGAGCGATATCTAAAGCCACCCCGGC 3') and subcloned into

120 pGEX-6p-1 plasmid vector (GE Healthcare) using BamHI and XhoI sites, resulting in the pGEX-6p-1-

121 NterGAFab plasmid and sequenced. To generate a BRET-based sensor construct containing N-terminal

122 regional along with tandem GAFab domain (NterGAFab), gene fragment encoding NterGAFab domain

123 was subcloned into the pGFP²-MCS-Rluc plasmid vector (PerkinElmer Life Sciences) using EcoRV

124 and $X$ hoI sites, resulting in the generation of pGFP $^{2}$-NterGAFab-Rluc plasmid.

\section{Expression and purification of the GAF domain constructs of CyaB2}

126 To express and purify GST fusion proteins, E. coli BL21 (DE3) cells were transformed with specific

127 plasmid and cells were induced using $100 \mu \mathrm{M} \mathrm{IPTG}$ at $37^{\circ} \mathrm{C}$ for $3 \mathrm{~h}$. Cells were collected by

128 centrifugation and cell pellet was resuspended in lysis buffer containing $50 \mathrm{mM}$ Tris $\left(\mathrm{pH} 8.2\right.$ at $\left.4{ }^{\circ} \mathrm{C}\right)$,

$129100 \mathrm{mM} \mathrm{NaCl}, 10 \%$ glycerol, $2 \mathrm{mM}$ PMSF, $1 \mathrm{mM}$ benzamidine. Cells were lysed by sonication and

130 lysate was centrifuged at $30,000 \mathrm{~g}$ for $30 \mathrm{~min}$ at $4{ }^{\circ} \mathrm{C}$. Supernatant was collected and interacted with

131 pre-equilibrated Glutathione Sepharose 4B beads (GE Healthcare Life Sciences) at $4{ }^{\circ} \mathrm{C}$ for $1 \mathrm{~h}$. Post

132 interaction, beads were washed with buffer containing $50 \mathrm{mM}$ Tris $\left(\mathrm{pH} 8.2\right.$ at $\left.4{ }^{\circ} \mathrm{C}\right), 100 \mathrm{mM} \mathrm{NaCl}$,

$1330.1 \%$ TritonX-100 followed by three washes with buffer containing $50 \mathrm{mM}$ Tris ( $\mathrm{pH} 8.2$ at $4{ }^{\circ} \mathrm{C}$ ), 100 
$134 \mathrm{mM} \mathrm{NaCl}, 10 \%$ glycerol. The protein-bound GSH beads were resuspended in buffer containing 25

$135 \mathrm{mM}$ HEPES, $100 \mathrm{mM} \mathrm{NaCl}$ and $10 \%$ glycerol and stored at $4^{\circ} \mathrm{C}$ till further use.

136 To express the His $_{6}-\mathrm{GAFb}$ protein, E. coli BL21DE3 cyc- cells were transformed with pPRO-Ex-B-

$137 \mathrm{GAFb}$ plasmid DNA and induced with $500 \mu \mathrm{M}$ IPTG for $3 \mathrm{~h}$ at $37^{\circ} \mathrm{C}$ (Nambi et al. 2010). Cells were

138 harvested by centrifugation at $6000 \mathrm{~g}$ for $20 \mathrm{~min}$ and the cell pellet was resuspended in lysis buffer [20

$139 \mathrm{mM}$ Tris- $\mathrm{HCl}(\mathrm{pH}$ 7.5), $100 \mathrm{mM} \mathrm{NaCl}, 5 \mathrm{mM} \beta$-mercaptoethanol, $5 \mathrm{mM}$ imidazole and EDTA free

140 protease inhibitor tablet (Roche)]. Cells were lysed by sonication and centrifuged at $17,000 \mathrm{~g}$ at $4{ }^{\circ} \mathrm{C}$

141 for $40 \mathrm{~min}$. The supernatant was collected and incubated with Talon resin (Invitrogen) at $4{ }^{\circ} \mathrm{C}$ for $1 \mathrm{~h}$.

142 The resin was then transferred into columns, and washed with lysis buffer and wash buffer (lysis buffer

143 with $10 \mathrm{mM}$ imidazole) followed by elution buffer containing lysis buffer with $150 \mathrm{mM}$ imidazole.

144 Further purification was achieved by size-exclusion chromatography on a Superdex 200 column using

145 the AKTA FPLC System (GE Healthcare Life Sciences).

\section{Cyclic nucleotide binding assays}

147 Cyclic nucleotide binding assays were carried out essentially as described earlier (Sopory et al. 2003)

148 using 1-5 $\mu \mathrm{g}$ of purified GST fusion proteins bound to glutathione beads in buffer containing $25 \mathrm{mM}$

149 HEPES, $100 \mathrm{mM} \mathrm{NaCl}, 10 \%$ glycerol and $200 \mu \mathrm{M}$ PMSF, in the presence of of 2,8 $\left[{ }^{3} \mathrm{H}\right]$-cAMP (

$150100,000 \mathrm{dpm} ; 28.1 \mathrm{Ci} / \mathrm{mmol}$; MPI Biomedicals) either in the absence or presence of unlabeled cAMP

151 or cGMP, in a total reaction volume of $50 \mu \mathrm{L}$. Reactions were incubated at $37{ }^{\circ} \mathrm{C}$ for $1 \mathrm{~h}$ and then

152 filtered through GF/C filters (Whatman), which were then washed with $6 \mathrm{~mL}$ of ice-cold washing

153 buffer (10 mM Tris, $\mathrm{pH} 7.5,100 \mathrm{mM} \mathrm{NaCl}$ and $10 \%$ glycerol). The filters were then dried and

154 radioactivity was measured in a liquid scintillation counter. 


\section{Docking of cyclic nucleotides to the GAFb domain of CyaB2}

156 Docking was performed using AutoDock (Version 3.0.5) (Morris 1998) implemented using AutoDock

157 Tools (Molecular Graphics Laboratory). The performance of AutoDock was tested by first docking

158 cAMP into the GAFb domain. For docking cNMPs to the GAFb domain of CyaB2, the atomic

159 structure comprising residues Leu270 to Leu431 of CyaB2 (PDB: 1YKD) was selected to be used as

160 the macromolecule (Martinez et al. 2005). All water molecules and cAMP were removed from the

161 structure before docking. Atomic coordinates of cAMP and cGMP were generated from the SMILES

162 structure descriptor format, available in the PubChem database, using Online SMILES Translator and

163 Structure File Generator (http://cactus.nci.nih.gov/services/translate/). Charges were added to the atoms

164 and the final structure file for AutoDock was prepared using the Dundee PRODRG2 Server

165 (http://davapc1.bioch.dundee.ac.uk/programs/prodrg/) (Schuttelkopf \& van Aalten 2004). Atomic

166 volume and solvation parameters were assigned to the protein molecule using default values. Polar

167 hydrogen atoms were added and Kollman charges were assigned using the built in function in

168 AutoDock Tools. Grids were used at a spacing of $0.375 \AA$ covering the cAMP binding site in a cube of

$16990 \times 90 \times 90$ grid points with the grid center placed near the phosphate group of cAMP as found in the

170 crystal structure. Docking was performed with a Lamarckian genetic algorithm with a total of 50

171 genetic algorithm runs for cAMP and 20 for cGMP. Other docking parameters were: population size $=$

17250 , mutation rate $=0.02$, crossover rate $=0.8$, number of genetic algorithm evaluations $=250000$,

173 number of genetic algorithm generations $=2700000$ and genetic algorithm elitism $=1$. Results were

174 analyzed using command get_docked and Pymol (The PyMOL Molecular Graphics System, Version

175 1.5.0.4 Schrödinger, LLC). 


\section{Cell culture and transfection}

177 Human embryonic kidney (HEK) 293T cells were maintained in Dulbecco's modified Eagle's media

178 (DMEM) with $10 \%$ fetal calf serum, $120 \mathrm{mg} / \mathrm{L}$ penicillin and $270 \mathrm{mg} / \mathrm{L}$ streptomycin at $37{ }^{\circ} \mathrm{C}$ in a $5 \%$

$179 \mathrm{CO}_{2}$ humidified incubator. Transfection was performed with polyethyleneimine lipid according to

180 manufacturers' protocols. Expression of proteins was monitored by Western Blot analysis using an

181 antibody raised in rabbit against the Rluc protein and described below.

\section{Generation of polyclonal antibody against Rluc}

183 Polyclonal antibodies against Rluc protein was raised in rabbits using $\mathrm{His}_{6}$-Rluc protein essentially as

184 described previously (Bakre et al. 2000b). Rluc gene was released from pRluc-N1 plasmid vector

185 (PerkinElmer Life Sciences) and subcloned into pPRO-Ex-C (Invitrogen) using BamHI and XbaI sites

186 to generate the pPRO-Ex-C-Rluc plasmid. To express the protein, E. coli BL21DE3 were transformed

187 with pPRO-Ex-C-Rluc plasmid and induced with $100 \mu \mathrm{M}$ IPTG. His ${ }_{6}$-Rluc protein formed inclusion

188 bodies. Aggregated protein was solubilized using urea and used for antibody generation. The primary

189 dose of immunogen $(\sim 500 \mu \mathrm{g})$ was in Freund's complete adjuvant and booster dose of immunogen

$190(\sim 250 \mu \mathrm{g})$ was in Freund's incomplete adjuvant. The presence of antibody was detected by ELISA and

191 Western Blot analysis.

\section{In vitro BRET assays}

193 All BRET assays were performed using the $\mathrm{BRET}^{2}$ assay components i.e. acceptor - $\mathrm{GFP}^{2}$, donor -

194 Rluc and Rluc substrate - Coelenterazine 400a. In vitro BRET assays were performed as described

195 previously (Biswas et al. 2008; Biswas \& Visweswariah 2011). HEK 293T cells transfected with

196 appropriate plasmids were lysed in a buffer of 50 mM HEPES (pH 7.5), containing 2 mM EDTA, 1

$197 \mathrm{mM}$ dithiothreitol, $100 \mathrm{mM} \mathrm{NaCl}, 10 \mathrm{mM}$ sodium pyrophosphate, $80 \mu \mathrm{M} \beta$-glycerophosphate, $1 \mathrm{mM}$ 
198 benzamidine, $1 \mu \mathrm{g} / \mathrm{mL}$ aprotinin, $1 \mu \mathrm{g} / \mathrm{mL}$ leupeptin, $5 \mu \mathrm{g} / \mathrm{mL}$ soybean trypsin inhibitor, $100 \mu \mathrm{M}$

199 sodium orthovanadate and $10 \%$ glycerol. Following brief sonication, the lysates was centrifuged at

$20013,000 \mathrm{~g}$ and the cytosol was collected. Aliquots of the cytosol were incubated with $1 \mathrm{mM} \mathrm{cNMP}$ in 201 buffer of $50 \mathrm{mM}$ HEPES, $\mathrm{pH} 7.5$, containing $100 \mathrm{mM} \mathrm{NaCl}$ in a total volume of $40 \mu \mathrm{L}$, at $37^{\circ} \mathrm{C}$ for 10

$202 \mathrm{~min}$. Coelenterazine 400a (Molecular Imaging Products) was added to a final concentration of $5 \mu \mathrm{M}$

203 and emissions were collected for $0.8 \mathrm{~s}$ in a Victor $^{3}$ microplate reader (Perkin Elmer). Emission filters

204 used for Rluc and GFP ${ }^{2}$ emission were $410 \mathrm{~nm}$ (bandpass $80 \mathrm{~nm}$ ) and $515 \mathrm{~nm}$ (bandpass $30 \mathrm{~nm}$ ),

205 respectively. BRET was calculated as the ratio of GFP emission per second to Rluc emission per

206 second, and the average of three such measurements is reported.

\section{Cellular BRET assays}

208 HEK 293T cells were transfected with pGFP²-GAFb-Rluc plasmid in $10 \mathrm{~cm}$ tissue culture dishes. Forty 209 eight hours post transfection, medium was removed, and monolayers treated with Dulbecco's

210 phosphate buffered saline containing $5 \mathrm{mM}$ EDTA for $5 \mathrm{~min}$ at $37^{\circ} \mathrm{C}$ in the incubator following which

211 the EDTA solution was removed, and cells resuspended in phenol-red free DMEM, containing 10\%

212 fetal calf serum. Cells $\left(\sim 10^{5}\right)$ were then treated with $100 \mu \mathrm{M}$ of either forskolin (for $5 \mathrm{~min}$ ) or sodium

213 nitroprusside (for $2 \mathrm{~min}$ ). BRET was determined as described above.

\section{Intracellular cNMP estimation}

215 Intracellular levels of cyclic nucleotide monophosphates (cNMP) were measured from the cells used

216 for BRET measurements, or parallely transfected and treated cells. Cells were lysed in $0.1 \mathrm{~N} \mathrm{HCl}$ and

217 cNMP was measured by radioimmunoassay as described earlier (Bakre et al. 2000a). 


\section{HDXMS of the GAFb domain of CyaB2}

219 The cAMP-free GAFb domain purified by size exclusion chromatography was concentrated to $50 \mu \mathrm{M}$ 220 using vivaspin concentrators (Sartorius Stedim Biotech GmbH, Goettingen, Germany). Samples were 221 prepared by adding $1 \mathrm{mM}$ cAMP or cGMP apo GAFb domain protein. $2 \mu \mathrm{L}$ each of apo, cAMP-, or 222 cGMP-bound GAFb domain in $20 \mathrm{mM}$ Tris- $\mathrm{HCl}(\mathrm{pH} 7.5), 100 \mathrm{mM} \mathrm{NaCl}$ and $5 \mathrm{mM}$ buffer were 223 diluted and incubated with $18 \mu \mathrm{L}$ of $\mathrm{D}_{2} \mathrm{O}(99.90 \%)$ (Fluka BioChemika, Buchs, Switzerland) resulting

224 in a final deuterium concentration of $90 \%$. Hydrogen-deuterium exchange was carried out at $20{ }^{\circ} \mathrm{C}$ for 225 various time points $(0.5,1,2,5$ and $10 \mathrm{~min})$. The exchange reaction was quenched by adding $40 \mu \mathrm{l}$ of 226 prechilled quench buffer (0.1\% trifluoroacetic acid (Fluka BioChemika, Buchs, Switzerland) to get a 227 final $\mathrm{pH}$ read of 2.5. An aliquot $(50 \mu \mathrm{l})$ of the quenched sample was then injected on to a chilled 228 nanoUPLC sample manager (beta test version, Waters, Milford, MA) as previously described 229 (Badireddy et al. 2011; Wales et al. 2008). Peptides were detected and sequenced and mass was 230 measured on a Synapt HDMS mass spectrometer (Waters, Manchester, UK) acquiring in the $\mathrm{MS}^{\mathrm{E}}$ 231 mode, a nonbiased, nonselective CID method (Bateman et al. 2002; Li et al. 2009; Shen et al. 2009; 232 Silva et al. 2005).

233 Sequence identifications were made from $\mathrm{MS}^{\mathrm{E}}$ data from undeuterated samples using ProteinLynx 234 Global Server 2.4 (beta test version) (Waters, Milford, MA) (Geromanos et al. 2009; Li et al. 2009) 235 and searched against sequence of GAFb domain with no enzyme specified and no modifications of 236 amino acids. Identifications were only considered if they appeared at least twice out of three replicate runs. The precursor ion mass tolerance was set at $<10 \mathrm{ppm}$ and fragment ion tolerance was set at $<20$

238 ppm. Only those peptides that satisfied the above criteria through Database search pass 1 were selected 239 and are listed in Table 1 (Li et al. 2009). The default criterion for false positive identification (Value = 240 4) was applied. These results showed that $\mathrm{MS}^{\mathrm{E}}$ data searched with PLGS 2.4 maximized identification 
241 of peptides and were used for deuterium exchange analysis. These identifications were mapped to

242 subsequent deuteration experiments using prototype custom software (HDX browser, Waters, Milford).

243 Data on each individual peptide at all periods were extracted using this software, and exported to HX-

244 Express (Weis et al. 2006) for analysis. A total number of 38 peptide fragments yielded primary

245 sequence coverage of $96 \%$. Continuous instrument calibration was carried out with Glu-fibrinogen

246 peptide at $100 \mathrm{fmol} / \mu \mathrm{l}$. We also visually analyzed the data to ensure only well resolved peptide isotopic

247 envelopes were subjected to quantitative analysis.

\section{Statistical analysis}

249 All experimental data were analyzed using GraphPad Prism and represent the mean \pm S.E.M.

\section{RESULTS}

\section{GAFb domain of CyaB2 binds both cAMP and cGMP}

252 GAF domains associated with enzymes such as nucleotide cyclases and phosphodiesterases are often 253 present in tandem repeats (Bruder et al. 2006; Schultz 2009). In the case of CyaB2, both the GAFa and $254 \mathrm{GAFb}$ domains bind cAMP. However, binding of cAMP to the GAFb domain is likely to trigger the 255 conformational changes in the protein that enhance adenylyl cyclase activity. We therefore tested if an 256 isolated $\mathrm{GAFb}$ domain of $\mathrm{CyaB} 2$ was able to bind ligand by direct radiolabeled cyclic nucleotide 257 binding assays. The isolated GAFb domain encompassing residues Leu 270 to Leu 431 fused to GST 258 was expressed in bacteria and purified. High affinity cAMP binding, with a $K_{\mathrm{D}}$ of $0.8 \pm 0.2 \mu \mathrm{M}$ (Fig. 259 1B \& C) was observed, and was similar to the $\mathrm{EC}_{50}$ value $(\sim 1.3 \mu \mathrm{M})$ reported previously from assays 260 monitoring cAMP-mediated activation of a related adenylyl cyclase domain (Bruder et al. 2005). This 261 result along with our previous studies using the isolated GAFa domain of PDE5 (Biswas et al. 2008) 
262 establishes that isolated GAF domains are able to bind their respective ligand even in the absence of 263 the second GAF domain, or other associated catalytic domains.

264 Previous studies have shown that the GAF domains associated with nucleotide cyclases and 265 phosphodiesterases show specificity in binding either cAMP or GMP. For instance, the GAFa domain 266 of PDE5 specifically binds cGMP (Biswas et al. 2008; Sopory et al. 2003), while the GAFb domain of 267 PDE2 binds cAMP (Martinez et al. 2002b). The tandem GAF domains of CyaB2 have also been shown 268 to be highly specific for cAMP as monitored by the activation of the CyaB1-CyaB2 fusion protein 269 (Bruder et al. 2005). However, we observed that cGMP could efficiently compete with cAMP for 270 binding to the isolated GAFb domain, with an $\mathrm{IC}_{50}$ of $7.6 \pm 1.9 \mu \mathrm{M}$ (Fig. 1C), in contrast to the 271 specificity of nucleotide-mediated activation of the adenylyl cyclase domain fused to the tandem GAF 272 domains of CyaB2 (Bruder et al. 2005).

273 To make a direct comparison of binding specificity, we performed cyclic nucleotide binding assays 274 with a construct containing both the GAF domains. For this, a GST fusion of the tandem GAFab 275 domains encompassing residues S77 to L431 was expressed and purified. Competition radiolabeled 276 nucleotide binding assays with cAMP and cGMP revealed that the tandem GAFab domain show much 277 higher affinity for cAMP ( $\mathrm{IC}_{50} 0.05 \pm 0.02 \mu \mathrm{M} ; 16$-fold higher affinity than the isolated GAFb domain) 278 and a much reduced affinity for cGMP ( $\mathrm{IC}_{50} 2651 \pm 870 \mu \mathrm{M} ; 348$-fold lower affinity compared to the 279 isolated GAFb domain) (Fig. 1D). Therefore, while the isolated GAFb domain showed only 10 -fold 280 selectivity towards cAMP, the tandem GAFab domain showed much higher ( $\sim 50,000$-fold) selectivity 281 for cAMP. Thus, although nucleotide binding is preserved in the isolated GAFb domain, removal of 282 the associated GAFa domain results in a reduction in both affinity and specificity of nucleotide 283 binding. 
284 In silico docking of cGMP to the GAFb domain of CyaB2

285 To gain insight into the mechanism by which cGMP could interact with the isolated GAFb domain, we 286 performed in silico docking of cGMP on the structure of the GAFb domain. We removed the bound 287 cAMP molecule from the crystal structure of the GAFb domain and used it as the receptor (Martinez et

We then performed mutational analysis to get further insights into ligand binding to the GAFb domain and validate the docking results. Analysis of the crystal structure of the GAFb domain showed an interaction between the side chain of I308 and the adenosine ring of cAMP (Martinez et al. 2005). An 
307 equivalent interaction is conserved in cyclic nucleotide binding GAF domains (Fig. 2C) but this

308 interaction should be dispensable if cGMP bound as predicted in mode 1. However, interaction of

309 cGMP with Thr 293 should be important for binding in mode 1 and could be dispensable for binding in

310 mode 2. Therefore, we mutated the Ile 308 and Thr 293 to A (I308A \& T293A mutants), and

311 performed radiolabeled ligand binding assays with the purified mutant GAFb domains. We observed

312 an expected $\sim 50 \%$ reduction in the binding of ${ }^{3} \mathrm{H}$-cAMP to the I308A mutant GAFb domain (Fig. 2D),

313 correlated with a significant reduction in the affinity for cAMP (Fig. 2E \& G). In contrast, the affinity

314 for cGMP remained unaltered in this mutant protein (Fig. 2F \& G). The T293A mutant protein,

315 however, showed a reduction in the affinity for cGMP (Fig. 2E \& G) but no change in the affinity of

316 cAMP binding (Fig. 2F \& G). Taken together, these results suggest that cGMP binds to the GAFb

317 domain as seen in mode 2. Additionally, unlike cAMP, cGMP binding does not require interaction with

318 Ile 308 , indicating subtle differences in residues interacting with the nucleotides.

\section{Ligand induced structural changes in the GAF domain are subtle and cannot be detected by}

\section{BRET}

321 Ligand binding to the GAF domains in CyaB2 is highly cooperative (Bruder et al. 2005) and acts as an 322 allosteric signal that results in the activation of the C-terminal adenylyl cyclase domain (Kanacher et 323 al. 2002). This implies that ligand binding to the GAFb domain may result in a structural change that is 324 communicated to both the N-terminal GAFa domain and the C-terminal adenylyl cyclase domain (Fig. 325 1A). We utilized BRET technology to determine if ligand binding to the GAFb domain alone results in 326 significant structural rearrangements. We have earlier used this strategy successfully to detect cGMP327 induced structural changes in the isolated GAFa domain of PDE5 (Biswas et al. 2008), as well as in the 328 full-length PDE5 (Biswas \& Visweswariah 2011). 
329 We generated a fusion protein containing $\mathrm{GFP}^{2}$ at the $\mathrm{N}$ - and Rluc protein to the C-terminal ends of

$330 \mathrm{GAFb}$ (GAFb sensor) (Fig. 3A). The GAFb sensor protein was expressed in HEK293T cells and

331 detected by western blot analysis using antibodies to Rluc (Fig. 3B). Lysates prepared from HEK293T

332 cells expressing the GAFb sensor were incubated in the absence or presence of $1 \mathrm{mM} \mathrm{cAMP}$ or cGMP, 333 and BRET was measured. We used the F163A mutant GAFa domain of PDE5, which binds both 334 cAMP and cGMP, for the purpose of comparison (Biswas et al. 2008). Basal BRET ratio of the GAFb 335 sensor was higher compared to the PDE5 GAFa(F163A) sensor (Fig. 3C), indicating that the GAFb 336 sensor expressed in mammalian cells is folded and could potentially bind ligand. Importantly, unlike 337 the PDE5 GAFa(F163A) sensor which showed a large increase in the BRET ratio in the presence of 338 both cAMP and cGMP, no change in the BRET ratio was observed for the GAFb sensor in the 339 presence of either cAMP or cGMP (Fig. 3C).

341 To rule out the requirement of any cellular factor for the induction of structural changes in the GAF 342 domain, we performed experiments with live cells expressing the GAFb sensor. Intracellular levels of 343 cAMP were elevated using forskolin, (Litosch et al. 1982), and intracellular cGMP levels were 344 increased by treating cells with sodium nitroprusside (SNP) (Murad 1986). Although both forskolin 345 and SNP treatment resulted in increased intracellular levels of cAMP and cGMP respectively, no 346 significant changes were observed in the BRET ratios (Fig. 3D \& 3E)

347 The lack of change in BRET may indicate that either there is no substantial structural change induced 348 in the GAFb domain on ligand binding, or the change in conformation induced by ligand binding could 349 not be detected due to lack of a specific structural element in the construct used for BRET (Russwurm 350 et al. 2007). We therefore generated fusion constructs containing either the tandem GAFab domains or 351 the tandem GAFab domains along with the complete N-terminal region of CyaB2 (called as GAFab 
352 and NterGAFab sensors, respectively). Expression levels of these proteins were lower than that of the 353 isolated GAFb domain (Fig. 3B), and the basal BRET of the constructs decreased in the order GAFb > 354 GAFab $>$ NterGAFab (Fig. 3C). This change in the basal BRET ratio suggested that we were able to 355 detect spatial positioning of GFP ${ }^{2}$ and Rluc in the sensor constructs. However, incubation of lysates 356 prepared from cells expressing either the GAFab or the NterGAFab sensor with cAMP or cGMP (1 $357 \mathrm{mM}$ ) did not result in a significant alteration in the BRET (Fig. 3C).

\section{Distinct changes in the dynamics of the GAFb domain of CyaB2 induced by cAMP and cGMP}

\section{9 binding}

360 The lack of observable change in the BRET of the GAF domains of CyaB2 on ligand binding was 361 intriguing. We therefore decided to monitor more subtle structural changes in the GAFb domain at a 362 higher resolution using amide hydrogen/deuterium exchange mass spectrometry (HDXMS). His $_{6^{-}}$ 363 tagged GAFb domain was expressed in bacteria and purified for use in these experiments. Complete 364 pepsin digestion of the protein under deuterium exchange quench conditions $(\mathrm{pH}=2.5)$ resulted in the 365 generation of multiple peptide fragments across the domain with $>90 \%$ sequence coverage (Fig. 4), 366 thus providing a detailed overview of the solvent accessibility and dynamics of the GAFb domain at 367 peptide resolution.

368 A comparison of amide exchange of various peptides in the absence and presence of cAMP showed a 369 decrease in exchange at the central core region comprising the ligand-binding pocket (Fig. 4A), 370 suggesting a 'closing' of the 'open' ligand binding pocket of the GAFb domain. These include peptide 371 (305-317) spanning parts of $\beta 1$ and $\beta 2$ sheets, and $\beta 1-\beta 2$ loop showed lower dynamics in the presence 372 of cAMP. The peptide (305-317) contains two residues that interact with cAMP, namely Ile 308 and 373 Thr 309. As discussed previously, Ile 308 provides hydrophobic stacking interaction to cAMP while 
374 Thr 309 interacts with the N6 of cAMP, forming H-bond through the peptide backbone carbonyl 375 oxygen.

376 Interestingly, cAMP binding resulted in an increased solvent accessibility of peptides arising from the $377 \mathrm{~N}$ - and C-terminal helices. The N-terminal $\alpha 2$ helix connects the GAFb domain to the GAFa domain, 378 and the C-terminal $\alpha 5$ helix connects the GAFb domain to the catalytic adenylyl cyclase domain of 379 CyaB2. This suggests that the structural changes observed here are signatures of allosteric signal 380 transduction from the GAFb domain to both the GAFa and the adenylyl cyclase domain. The absence 381 of any change in the BRET signal observed with the GAFb sensor construct indicates that the increase 382 in amide exchange following cAMP binding arose from an increase in the entropy of these parts of the $383 \mathrm{GAFb}$ domain, and not as a consequence of a gross change in the relative structure of the protein.

384 Binding of cGMP, on the other hand, resulted in remarkably less changes in the amide exchange of the $385 \mathrm{GAFb}$ domain, and only some regions in the ligand-binding pocket showed an increase in exchange 386 compared to the unliganded protein (Fig. 4B). A comparison of the exchange profile of the GAFb 387 domain in the presence of cAMP and cGMP clearly showed a number of differences (Fig. 4C), 388 especially in the region containing peptide (305-317) harboring the residue I308. This is in agreement 389 with direct cyclic nucleotide ligand binding data, and confirmed that indeed cAMP and cGMP bind to 390 the GAFb domain in distinct modes. In addition, a lack of alteration in the dynamics of the terminal 391 helices in the presence of cGMP provides a structural basis for the lack of allosteric regulation induced 392 by cGMP binding to the GAFb domain (Bruder et al. 2005).

\section{DISCUSSION}

394 Most cyclic nucleotide binding GAF domains are specific in terms of ligand binding. Efforts have been 395 directed towards understanding the mechanism by which these domains achieve specificity. Mutational 
396 and biochemical analysis of GAF domains from other proteins have provided some understanding of 397 the mechanism by which these structurally similar domains discriminate nucleotides (Linder et al. 398 2007; Schultz 2009). We propose that in addition to the specific interaction of certain residues with the 399 chemical groups present in nucleotides, the hydrophobic interaction provided by residue equivalent to 400 I308 help GAF domains in selecting a specific cyclic nucleotide. Mutations equivalent to I308A in the 401 GAFa domain of PDE5 (Sopory et al. 2003) and the GAFb domain of PDE2 (Wu et al. 2004) has been 402 403 shown to reduce cGMP affinity, while affinity for cAMP were reported to be largely unaffected. The GAFa domain of PDE5 and the GAFb domain of PDE2 are known to bind cGMP with high affinity while the affinity of these GAF domains is much less for cAMP. Thus, it appears that the I308 residue in cyclic nucleotide-binding GAF domains dictates the binding of the high affinity ligand. Importantly, in addition, we show here the important involvement of T293 in the GAFb domain of CyaA in binding cGMP (Fig. 2GC).

The crystal structure of the cAMP-bound GAFb domain showed that cAMP is largely buried, leading to the speculation that the ligand binding pocket is initially present in an open conformation ready to receive the ligand (Martinez et al. 2005). Reduction in the dynamics of peptides spanning the ligand411 binding pocket (helix $\alpha 4$, helix $\alpha 3$ and sheet $\beta 3$ ) in the presence of cAMP confirms that the domain 412 'closes' on ligand binding. A similar mechanism has been proposed for the GAFa domain of PDE5 on 413 binding cGMP (Wang et al. 2010). Interestingly, it appears that the way in which the 'open' GAF 414 domain 'closes' following binding of either the low affinity or high affinity ligand is different. This 415 kind of structural adaptation could not only be necessary to avoid steric hindrance while retaining 416 interactions between the GAF domain and the ligand, but could also play a role in the way the signal of 417 ligand binding to the GAF domain allosterically regulates the C-terminal catalytic domain. 
418 In addition to the mechanism of ligand specificity by a specific residue in the ligand-binding site, 419 comparison of ligand binding to the isolated GAFb domain and the tandem GAF domains revealed a 420 much higher degree of ligand selectivity. This indicates that the isolated GAFb domain and the tandem

421 GAF domains are structurally and biochemically different. Proteins exist in an ensemble of

422 conformations at steady state. The presence of the second GAF domain could influence ligand-binding 423 behavior of the associated GAFb domain by establishing new steady state conformations, allowing 424 concomitant ligand binding specificity, coupled with precise ligand-induced allosteric regulation of 425 these proteins.

\section{CONCLUSION}

427 Our results provide insights on the basis of nucleotide selectivity and proximal conformational changes 428 that occur following cAMP binding to the GAFb domain of CyA2. They may also allow a molecular 429 understanding of the 'regulated unfolding' that needs to occur during activation of the C-terminal 430 catalytic domains associated with the GAF domains (Schultz \& Natarajan 2013), and also provide a 431 foundation for the design of molecules that could modulate GAF domain function and action.

\section{AUTHOR CONTRIBUTION}

434 All authors designed experiments. KHB, SB and AR performed experiments. KHB, GSA and SSV 435 analyzed and interpreted data, and wrote the paper.

\section{FUNDING SOURCES}

438 Funding has been provided by the Department of Biotechnology and Science and Technology (SSV), 439 and a fellowship from the Council for Scientific and Industrial Research (KHB), Government of India. 440 Support was also provided by the Mechanobiology Institute, National University of Singapore (NUS), 
441 Singapore and Waters Center of Innovation Program (to GSA). Personnel exchanges between

442 laboratories was funded by the NUS-India Research Initiative. KHB is currently supported by a

443 Research Fellowship from the Mechanobiology Institute, NUS, Singapore. 
Anantharaman V, Koonin EV, and Aravind L. 2001. Regulatory potential, phyletic distribution and evolution of ancient, intracellular small-molecule-binding domains. J Mol Biol 307:1271-1292.

Aravind L, Mazumder R, Vasudevan S, and Koonin EV. 2002. Trends in protein evolution inferred from sequence and structure analysis. Curr Opin Struct Biol 12:392-399.

Aravind L, and Ponting CP. 1997. The GAF domain: an evolutionary link between diverse phototransducing proteins. Trends Biochem Sci 22:458-459.

Badireddy S, Yunfeng G, Ritchie M, Akamine P, Wu J, Kim CW, Taylor SS, Qingsong L, Swaminathan K, and Anand GS. 2011. Cyclic AMP analog blocks kinase activation by stabilizing inactive conformation: conformational selection highlights a new concept in allosteric inhibitor design. Mol Cell Proteomics 10:M110 004390.

Bakre MM, Ghanekar Y, and Visweswariah SS. 2000a. Homologous desensitization of the human guanylate cyclase $\mathrm{C}$ receptor. Cell-specific regulation of catalytic activity. Eur J Biochem 267:179-187.

Bakre MM, Sopory S, and Visweswariah SS. 2000b. Expression and regulation of the cGMP-binding, cGMP-specific phosphodiesterase (PDE5) in human colonic epithelial cells: role in the induction of cellular refractoriness to the heat-stable enterotoxin peptide. $J$ Cell Biochem $77: 159-167$.

Bateman RH, Carruthers R, Hoyes JB, Jones C, Langridge JI, Millar A, and Vissers JP. 2002. A novel precursor ion discovery method on a hybrid quadrupole orthogonal acceleration time-of-flight (Q-TOF) mass spectrometer for studying protein phosphorylation. J Am Soc Mass Spectrom $13: 792-803$. 
468 Biswas KH, Sopory S, and Visweswariah SS. 2008. The GAF domain of the cGMP-binding, cGMP-

469

470

471

472

473

474

475

476

477

478

479

480

481

482

483

484 485

486

487

488

489

490 specific phosphodiesterase (PDE5) is a sensor and a sink for cGMP. Biochemistry 47:35343543.

Biswas KH, and Visweswariah SS. 2011. Distinct allostery induced in the cyclic GMP-binding, cyclic GMP-specific phosphodiesterase (PDE5) by cyclic GMP, sildenafil, and metal ions. J Biol Chem 286:8545-8554.

Bruder S, Linder JU, Martinez SE, Zheng N, Beavo JA, and Schultz JE. 2005. The cyanobacterial tandem GAF domains from the cyaB2 adenylyl cyclase signal via both cAMP-binding sites. Proc Natl Acad Sci U S A 102:3088-3092.

Bruder S, Schultz A, and Schultz JE. 2006. Characterization of the tandem GAF domain of human phosphodiesterase 5 using a cyanobacterial adenylyl cyclase as a reporter enzyme. $J$ Biol Chem 281:19969-19976.

Charbonneau H, Prusti RK, LeTrong H, Sonnenburg WK, Mullaney PJ, Walsh KA, and Beavo JA. 1990. Identification of a noncatalytic cGMP-binding domain conserved in both the cGMPstimulated and photoreceptor cyclic nucleotide phosphodiesterases. Proc Natl Acad Sci U S A 87:288-292.

Finn RD, Mistry J, Tate J, Coggill P, Heger A, Pollington JE, Gavin OL, Gunasekaran P, Ceric G, Forslund K, Holm L, Sonnhammer EL, Eddy SR, and Bateman A. 2010. The Pfam protein families database. Nucleic Acids Res 38:D211-222.

Geromanos SJ, Vissers JP, Silva JC, Dorschel CA, Li GZ, Gorenstein MV, Bateman RH, and Langridge JI. 2009. The detection, correlation, and comparison of peptide precursor and product ions from data independent LC-MS with data dependant LC-MS/MS. Proteomics 9:1683-1695. 
491 Heikaus CC, Pandit J, and Klevit RE. 2009. Cyclic nucleotide binding GAF domains from

492 phosphodiesterases: structural and mechanistic insights. Structure 17:1551-1557.

Heikaus CC, Stout JR, Sekharan MR, Eakin CM, Rajagopal P, Brzovic PS, Beavo JA, and Klevit RE. 2008. Solution structure of the cGMP binding GAF domain from phosphodiesterase 5: insights into nucleotide specificity, dimerization, and cGMP-dependent conformational change. J Biol Chem 283:22749-22759.

Kanacher T, Schultz A, Linder JU, and Schultz JE. 2002. A GAF-domain-regulated adenylyl cyclase from Anabaena is a self-activating cAMP switch. Embo J 21:3672-3680.

Li GZ, Vissers JP, Silva JC, Golick D, Gorenstein MV, and Geromanos SJ. 2009. Database searching and accounting of multiplexed precursor and product ion spectra from the data independent analysis of simple and complex peptide mixtures. Proteomics 9:1696-1719.

Linder JU, Bruder S, Schultz A, and Schultz JE. 2007. Changes in purine specificity in tandem GAF chimeras from cyanobacterial cyaB1 adenylate cyclase and rat phosphodiesterase 2. Febs J 274:1514-1523.

Litosch I, Hudson TH, Mills I, Li SY, and Fain JN. 1982. Forskolin as an activator of cyclic AMP accumulation and lipolysis in rat adipocytes. Mol Pharmacol 22:109-115.

Martinez SE, Beavo JA, and Hol WG. 2002a. GAF domains: two-billion-year-old molecular switches that bind cyclic nucleotides. Mol Interv 2:317-323.

Martinez SE, Bruder S, Schultz A, Zheng N, Schultz JE, Beavo JA, and Linder JU. 2005. Crystal structure of the tandem GAF domains from a cyanobacterial adenylyl cyclase: modes of ligand binding and dimerization. Proc Natl Acad Sci U S A 102:3082-3087.

Martinez SE, Wu AY, Glavas NA, Tang XB, Turley S, Hol WG, and Beavo JA. 2002b. The two GAF domains in phosphodiesterase 2A have distinct roles in dimerization and in cGMP binding. Proc Natl Acad Sci U S A 99:13260-13265. 
515 Morris GM, Goodsell, D. S., Halliday, R.S., Huey, R., Hart, W. E., Belew, R. K. and Olson, A. J. 1998. Automated Docking Using a Lamarckian Genetic Algorithm and and Empirical Binding Free Energy Function. J Computational Chemistry 19:1639-1662.

Murad F. 1986. Cyclic guanosine monophosphate as a mediator of vasodilation. J Clin Invest 78:1-5.

519 Nambi S, Basu N, and Visweswariah SS. 2010. cAMP-regulated protein lysine acetylases in mycobacteria. J Biol Chem 285:24313-24323.

Pandit J, Forman MD, Fennell KF, Dillman KS, and Menniti FS. 2009. Mechanism for the allosteric regulation of phosphodiesterase $2 \mathrm{~A}$ deduced from the X-ray structure of a near full-length construct. Proc Natl Acad Sci U S A 106:18225-18230.

Russwurm M, Mullershausen F, Friebe A, Jager R, Russwurm C, and Koesling D. 2007. Design of fluorescence resonance energy transfer (FRET)-based cGMP indicators: a systematic approach. Biochem J 407:69-77.

Russwurm M, Schlicker C, Weyand M, Koesling D, and Steegborn C. 2011. Crystal structure of the GAF-B domain from human phosphodiesterase 5. Proteins 79:1682-1687.

Schultz J. 2009. Structural and Biochemical Aspects of Tandem GAF Domains. In: Schmidt HHW, Hofmann F, and Stasch J-P, eds. cGMP: Generators, Effectors and Therapeutic Implications: Springer Berlin Heidelberg, 93-109.

Schultz JE, and Natarajan J. 2013. Regulated unfolding: a basic principle of intraprotein signaling in modular proteins. Trends Biochem Sci 38:538-545.

Schuttelkopf AW, and van Aalten DM. 2004. PRODRG: a tool for high-throughput crystallography of protein-ligand complexes. Acta Crystallogr D Biol Crystallogr 60:1355-1363.

Shen Z, Li P, Ni RJ, Ritchie M, Yang CP, Liu GF, Ma W, Liu GJ, Ma L, Li SJ, Wei ZG, Wang HX, and Wang BC. 2009. Label-free quantitative proteomics analysis of etiolated maize seedling leaves during greening. Mol Cell Proteomics 8:2443-2460. 
539 Shenoy AR, and Visweswariah SS. 2003. Site-directed mutagenesis using a single mutagenic oligonucleotide and DpnI digestion of template DNA. Anal Biochem 319:335-336.

541 Silva JC, Denny R, Dorschel CA, Gorenstein M, Kass IJ, Li GZ, McKenna T, Nold MJ, Richardson K, 542 Young P, and Geromanos S. 2005. Quantitative proteomic analysis by accurate mass retention time pairs. Anal Chem 77:2187-2200.

Sopory S, Balaji S, Srinivasan N, and Visweswariah SS. 2003. Modeling and mutational analysis of the GAF domain of the cGMP-binding, cGMP-specific phosphodiesterase, PDE5. FEBS Lett 539:161-166.

Wales TE, Fadgen KE, Gerhardt GC, and Engen JR. 2008. High-speed and high-resolution UPLC separation at zero degrees Celsius. Anal Chem 80:6815-6820.

Wang H, Robinson H, and Ke H. 2010. Conformation changes, N-terminal involvement, and cGMP signal relay in the phosphodiesterase-5 GAF domain. J Biol Chem 285:38149-38156.

Weis DD, Engen JR, and Kass IJ. 2006. Semi-automated data processing of hydrogen exchange mass spectra using HX-Express. J Am Soc Mass Spectrom 17:1700-1703.

Wu AY, Tang XB, Martinez SE, Ikeda K, and Beavo JA. 2004. Molecular determinants for cyclic nucleotide binding to the regulatory domains of phosphodiesterase 2A.J Biol Chem 279:3792837938.

Zoraghi R, Corbin JD, and Francis SH. 2004. Properties and functions of GAF domains in cyclic nucleotide phosphodiesterases and other proteins. Mol Pharmacol 65:267-278. 
Figure $\mathbf{1}_{\text {(on next page) }}$

Isolated GAFb domain binds both cAMP and cGMP

[A] Cartoon representation of the structure of the GAFb domain illustrating various secondary structure elements and bound cAMP molecule [PDB: 1YKD ( Martinez et al. 2005) ]. [B] Proteins $(\sim 1 \mu \mathrm{g})$ bound to glutathione beads were incubated with ${ }^{3} \mathrm{H}-\mathrm{CAMP}(\sim 1 \mathrm{nM})$ in the absence or presence of $10 \mu \mathrm{M}$ unlabeled cAMP. Data shown is a representative of assays performed thrice in duplicates, and values shown are mean $\pm \mathrm{S}$. E. M. The inset shows a Coomassie stained gel picture of the purified proteins used in the assay. [C] \& [D] Purified GST-GAFb [C] or GST-GAFab [D] proteins bound to beads were incubated with $\sim 1 \mathrm{nM}{ }^{3} \mathrm{H}$ CAMP and increasing concentration of unlabeled CAMP or CGMP. Data shown is mean $\pm \mathrm{S}$. E. $M$ of duplicate determinations and is representative of independent assays. 
A

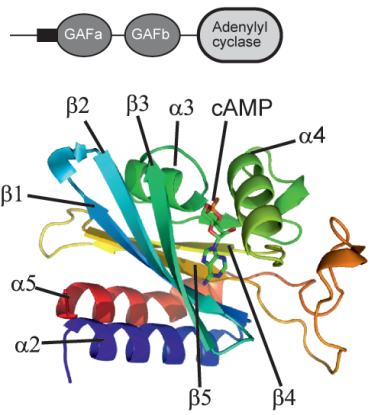

C

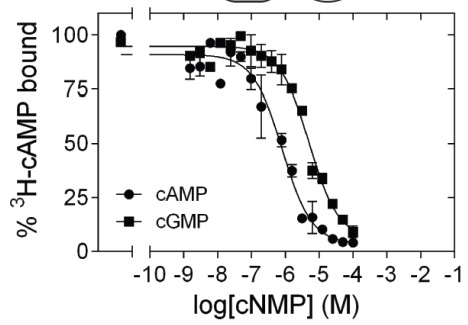

B

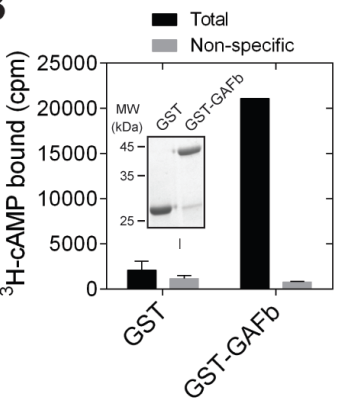

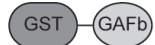

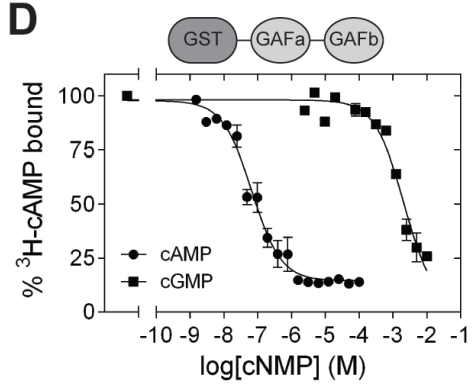




\section{Figure 2 (on next page)}

Ligand binding to the isolated GAFb domain of CyaB2

[A] Cartoon representation of the GAFb domain [PDB: 1YKD ( Martinez et al. 2005) ] with docked CAMP and CGMP conformers. Insets show zoomed in view of the ligand binding pocket of GAFb with docked cAMP (upper panel) and two different clusters of docked cGMP conformers indicated as CGMP-1 and CGMP-2 (lower panel). Side chains of T293 and I308 are also shown along with their interaction with the docked ligands. [B] Distribution of docked cGMP conformers obtained clustering with an RMSD of $0.5 \AA$. Mode 1 represents the cluster with maximum number of conformers and with lowest energy while mode 2 represents the cluster with CGMP docked in a way that mimics bound CAMP. Third cluster consisting of one conformer out of 20 was docked outside the ligand-binding pocket and therefore, has not been shown. [C] Conserved interaction between high affinity ligand, either CAMP or CGMP, with the residue equivalent to 1308 in different cyclic nucleotide binding GAF domains (GAFb domain of CyaB2 - CAMP [PDB: 1YKD]; GAFb domain of PDE2 - cGMP [PDB: 1MCO]; GAFa domain of PDE5 - cGMP [PDB: 2K31]; GAFb domain of PDE10 - CAMP [PDB: 2E4S]; GAFa domain of PDE6 - cGMP [PDB: 3DBA]. [D] Wild type (WT), I308A or T293A mutant GST-fusion proteins $(\sim 1-5 \mu \mathrm{g})$ bound to glutathione beads were incubated with ${ }^{3} \mathrm{H}-\mathrm{CAMP}(\sim 1 \mathrm{nM})$ in the absence or presence of $10 \mu \mathrm{M}$ unlabeled cAMP. Data shown is a representative of assays performed at least twice in duplicate, and values shown are mean \pm S. E. M. for $1 \mu$ g protein. The inset shows a Coomassie stained gel picture of the purified proteins used in the assay.

[E] \& [F] Purified wild type, 1308A or T293A mutant GST fusion proteins bound to beads were incubated with $\sim 1 \mathrm{nM}{ }^{3} \mathrm{H}-\mathrm{CAMP}$ and increasing concentration of unlabeled CAMP [E] or CGMP $[\mathbf{F}$ ]. Data shown is mean $\pm \mathrm{S}$. E. M of duplicate determinations and is representative of independent assays. [G] Log values of the $\mathrm{IC}_{50}$ obtained for the wild type, 1308A or T293A mutant proteins with either CAMP or CGMP are plotted. Data shown is mean \pm S. E. M. of $\mathrm{IC}_{50}$ values determined from multiple independent assays. 

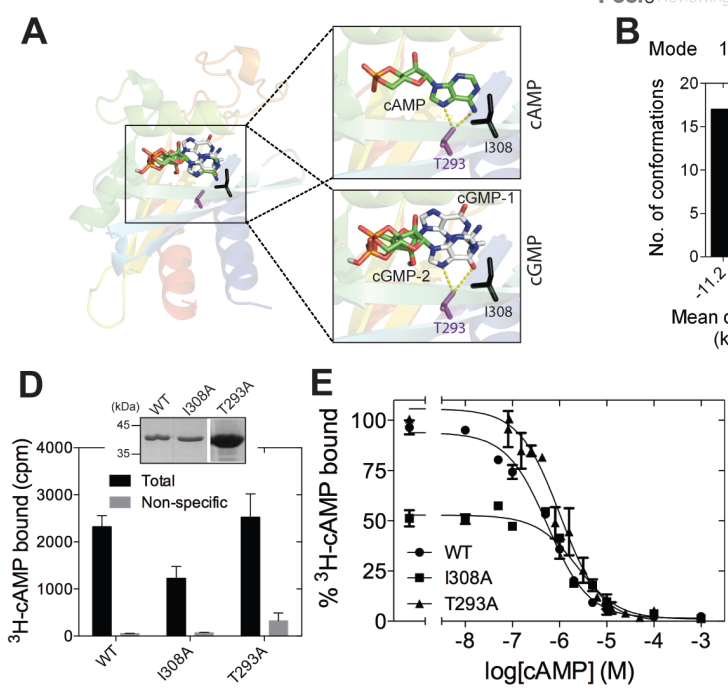

Peef reviewing PDF | [2014:11:3104:1:1:-NEW 13 Mar 2015]

\section{C}

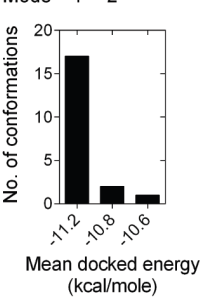

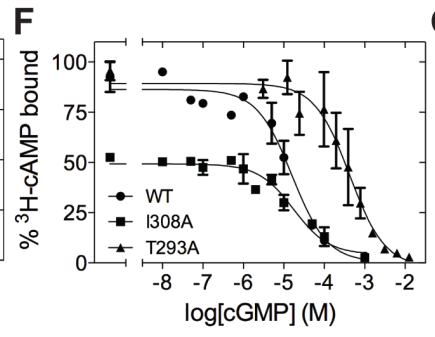

G

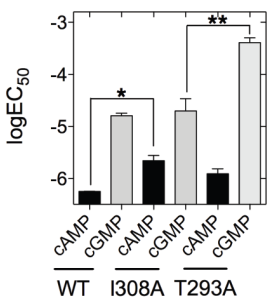




\section{Figure $\mathbf{3}$ (on next page)}

Ligand binding to the GAFb domain does not result in structural changes at the $\mathrm{N}$ - and C-termini

[A] Diagrammatic representation of various BRET-based sensor constructs used in the study.

[B] Western blot analysis using anti-Rluc polyclonal antibody to confirm the expression of GAFb, GAFab and NterGAFab sensor constructs. Expected molecular weight of the GAFb, GAFab and NterGAFab sensor constructs are 82.7, 103.8 and $112.7 \mathrm{kDa}$, respectively. [C] Lysates prepared from cells expressing the PDE5 GAFa(F163A), GAFb, GAFab and NterGAFab sensor constructs were incubated in the absence or presence of $1 \mathrm{mM}$ CAMP or CGMP at $37^{\circ} \mathrm{C}$ for 10 min followed by BRET measurement. [D \& E] HEK293T cells transfected with the GAFb sensor were treated with $100 \mu \mathrm{M}$ of either forskolin $(5 \mathrm{~min})$ [D] or SNP (2 min) [E] at $37^{\circ} \mathrm{C}$ and BRET was determined. Intracellular levels of CAMP or CGMP were determined using a parallel set of cells. Data shown are mean \pm S.E.M from a representative experiment performed in triplicate. 
A

PeerJ Reviewing Manuscript
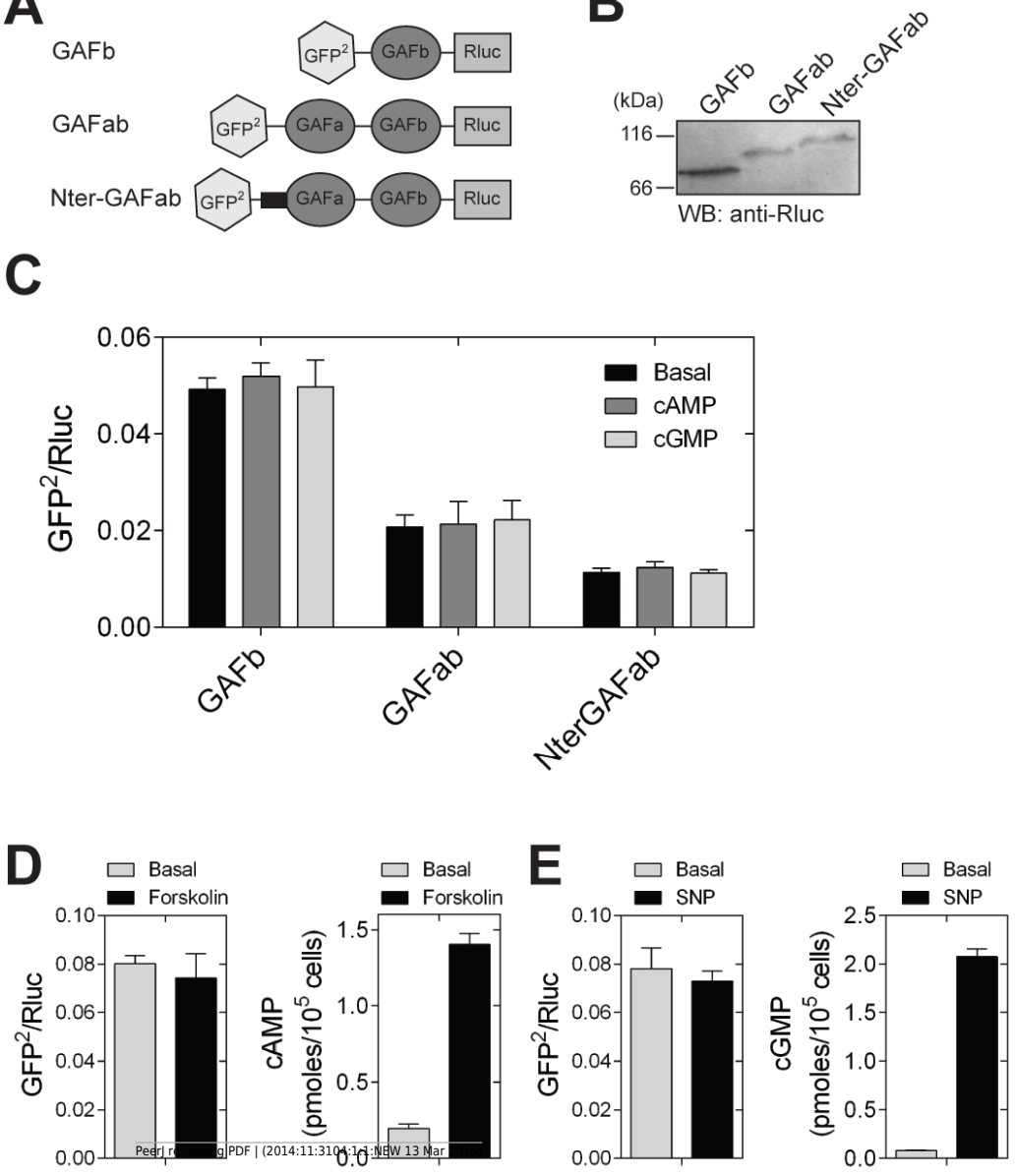


\section{Figure $\mathbf{4}$ (on next page)}

Protein-wide overview of structural changes induced in the GAFb domain of CyaB2 on ligand binding

Absolute difference in numbers of deuterons (inferred from difference in mass in Daltons ( $\mathrm{Da}$ ) ( $y$ axis) between free and CAMP-bound (top), free and CGMP-bound (middle) and CAMP- and cGMP-bound (bottom) states is plotted for each pepsin digest fragment listed from the $\mathrm{N}$ - to C-terminus ( $x$ axis) of GAFb domain for each deuterium exchange time point in a difference plot. Time $=0.5 \mathrm{~min}$ (orange), $2 \mathrm{~min}$ (red), $5 \mathrm{~min}$ (blue) and $10 \mathrm{~min}$ (black). Shifts in the positive scale represent increases in deuterium exchange and shifts in the negative scale represent decreases in deuterium exchange. A difference of $0.5 \mathrm{Da}$ is considered significant (dashed red line). Plots were generated using the software DYNAMX (Ver. 2.0 Waters). Each point represents a pepsin digest fragment. 
-PeeldRewiewing-M-mpruseript-

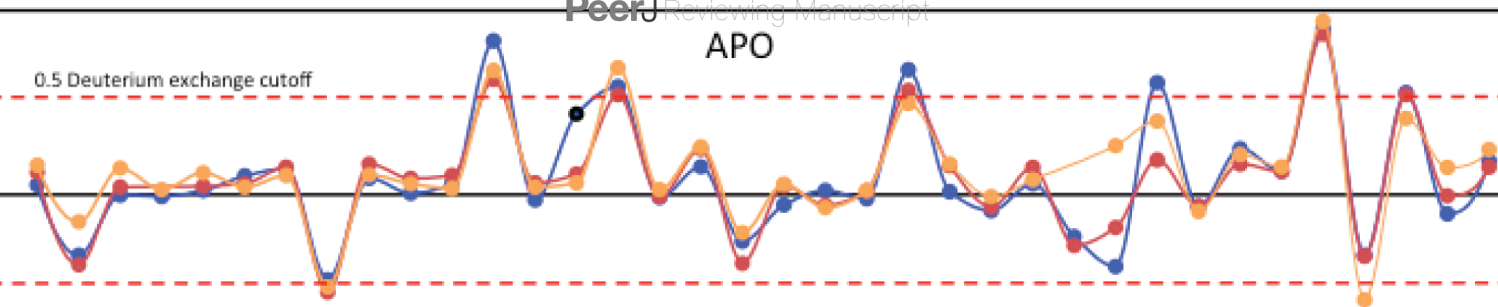

CAMP

- 0 \%

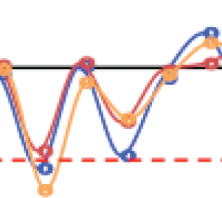

ses

\section{cGMP}

0.5 Deuterium exchange cutoff

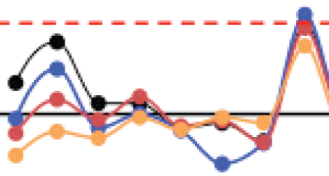

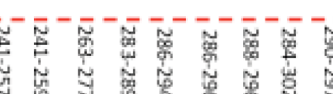

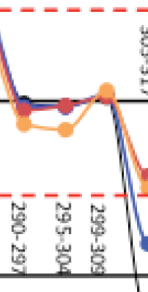

\section{CAMP}
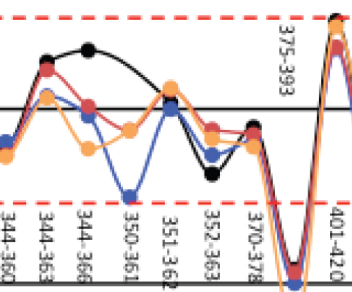

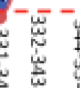
品 